\title{
Targeted treatment of imatinib-resistant chronic myeloid leukemia: Focus on dasatinib
}

This article was published in the following Dove Press journal:

OncoTargets and Therapy

22 April 2009

Number of times this article has been viewed

\section{Charles Chuah' \\ Junia $\mathrm{V}$ Melo $^{2}$}

'Singapore General Hospital and Duke-NUS Graduate Medical School, Singapore; ${ }^{2}$ Institute of Medical and Veterinary Science, South Australia, Australia
Correspondence: Charles Chuah Department of Haematology, Singapore General Hospital, Cancer and Stem Cell Biology Program, Duke-NUS Graduate Medical School, Singapore 169608

$\mathrm{Tel}+6563214595$

Fax +6562206210

Email charles.chuah.t.h@sgh.com.sg
Abstract: The efficacy of imatinib in chronic myeloid leukemia has been remarkable, but the development of resistance and the persistence of minimal residual disease have dampened the initial enthusiasm for this much heralded 'magic bullet'. Much progress has been made in elucidating the mechanisms which underlie imatinib resistance. The most common cause of such drug resistance is the selection of leukemic clones with point mutations in the Abl kinase domain leading to amino acid substitutions which prevent the appropriate binding of the drug. Other mechanisms include genomic amplification of $B C R-A B L$ and modulation of drug efflux or influx transporters. Dasatinib is a multi-target kinase inhibitor which has increased potency and is able to inhibit most Bcr-Abl mutant cell lines. Clinical trials of dasatinib in imatinib-resistant and -intolerant chronic myeloid leukemia and Philadelphia chromosome-positive acute lymphoid leukemia have shown that it is effective and well tolerated. In this review, we will discuss the pre-clinical development of dasatinib, the clinical trial data demonstrating its efficacy and tolerability and highlight certain aspects of its toxicity profile and mechanisms of resistance.

Keywords: drug resistance, tyrosine kinase inhibitors, Bcr-Abl

\section{Introduction}

Imatinib has, without doubt, been a major achievement for the treatment of chronic myeloid leukemia (CML), but resistance to this drug has become and will continue to be a therapeutic challenge. In early chronic phase (CP-CML) patients treated with imatinib at diagnosis, $18 \%$ failed to achieve a complete cytogenetic response (CCyR) after a median follow-up of five years while $6 \%$ progressed to the advance phase and $8 \%$ had a cytogenetic or hematologic relapse. ${ }^{1}$ Among CP-CML patients who had failed prior interferon treatment, $43 \%$ did not achieve a CCyR after a median duration of imatinib treatment of 65 months and 39\% progressed to the advance phase. ${ }^{2}$ Not surprisingly, in advanced phase patients treated with imatinib, resistance or relapse occurred in 75\% of accelerated phase (AP-CML) and 95\% of myeloid blast crisis (MBC-CML) patients. ${ }^{3}$ Single agent therapy with imatinib may not be the best longterm option in CML, at least for a proportion of patients, and other strategies need to be explored. Many novel compounds are currently being investigated pre-clinically and clinically, and therapeutic approaches to circumvent the problem of imatinibresistance are now possible. Dasatinib (BMS-354825, Sprycel; Bristol-Myers Squibb, New York, NY) and other Bcr-Abl tyrosine kinase inhibitors (TKI), such as nilotinib (AMN107, Tasigna; Novartis, Basel, Switzerland) and bosutinib (SKI-606; Wyeth, Madison, NJ) represent the newer generation of TKIs which have been shown to be effective and safe in imatinib-resistant and -intolerant CML patients. 


\section{Pre-clinical development of dasatinib}

The disruption of the proto-oncogene Src is associated with the pathogenesis of human cancers. ${ }^{4}$ Several synthetic small molecule inhibitors of Src-family kinases (SFK) have been developed, such as PD180970, AP23464, CGP76030, dasatinib, and bosutinib. These compounds also inhibit Bcr-Abl, Kit and platelet-derived growth factor receptor $\beta$ (PDGFR $\beta$ ), and have in vitro antiproliferative activity in imatinib-sensitive and -resistant CML cells. ${ }^{5-10}$

Dasatinib was identified among a series of novel 2-(aminopyridyl)-and 2-(aminopyrimidinyl)-thiazole5 -carboxamides as a compound with broad spectrum antiproliferative activity against hematological and solid tumor cell lines, and favorable pharmacokinetics after oral dosing. ${ }^{11} \mathrm{X}$-ray crystallographic studies of the threedimensional structure of Abl kinase complexed with dasatinib revealed that the compound binds to the activation loop of $\mathrm{Abl}$ in an active conformation. ${ }^{11}$ In addition, interactions between the Abl ATP-binding site and dasatinib were less critical for binding, suggesting that the compound would be active against the mutant kinase forms. ${ }^{11}$ Initial studies revealed that dasatinib is a potent multi-target kinase inhibitor of Bcr-Abl, SFK (Src, Lck, and Yes), PDGFR $\beta$ and Kit at nanomolar to sub-nanomolar concentrations (Table 1). ${ }^{11}$ Recently, dasatinib was also shown to bind to other tyrosine and serine/threonine kinases, such as the TEC family kinases, BTK and TEC, the mitogen-activated protein kinases, the receptor tyrosine kinase, discoidin domain receptor 1 and the ephrin receptors. ${ }^{12}$

Biochemical assays using purified GST-Abl kinase and cellular proliferation and Bcr-Abl tyrosine phosphorylation assays revealed that dasatinib was approximately 325 times

Table I Kinase profile of dasatinib

\begin{tabular}{ll}
\hline Kinase & ${\text { Enzyme } \mathbf{I C}_{50}, \mathbf{n M}}^{\text {B }}$ \\
\hline Bcr-Abl & $<1.0$ \\
Src & 0.5 \\
Lck & 0.4 \\
Yes & 0.4 \\
C-kit & 5 \\
PDGFR $\beta$ & 28 \\
P38 & 100 \\
Herl & 180 \\
Her2 & 710 \\
FGFR-I & 880 \\
MEK & 1700 \\
\hline
\end{tabular}

more potent than imatinib. ${ }^{13}$ Dasatinib was also able to inhibit the cellular proliferation, peptide substrate and Bcr-Abl tyrosine phosphorylation of all Bcr-Abl mutants at nanomolar concentrations with the exception of the T315I mutant. ${ }^{13}$ In addition, in vivo studies in murine models demonstrated the activity of dasatinib in inhibiting the leukemic cell growth and prolonging the survival of mice harboring wild type BcrAbl and the M351T, but not the T315I mutant. ${ }^{9}$

\section{Clinical efficacy of dasatinib}

Based on the promising pre-clinical data, clinical trials were embarked upon to test the efficacy and safety of dasatinib in patients who were resistant to or intolerant of imatinib.

\section{Phase I study}

CA180002 was a dose-escalation study for patients with imatinib-resistant or -intolerant CML or Philadelphia-positive acute lymphoblastic leukemia $(\mathrm{Ph}+\mathrm{ALL}) \cdot{ }^{14} \mathrm{~A}$ total of 84 patients were enrolled into the study. Dasatinib was administered at total daily doses ranging from 15 to $240 \mathrm{mg}$, once or twice daily. Dasatinib was generally well tolerated. Grade 3 or 4 hematologic suppression was common especially in the advanced phases, resulting in treatment interruption in $60 \%$ and dose reduction in $25 \%$ of patients. Nonhematologic toxicities included pleural effusion, diarrhea, nausea, vomiting, gastrointestinal hemorrhage, peripheral or periorbital edema, pericardial effusion, pulmonary edema, rash, flushing headache, fatigue, and tumor lysis syndrome. Most of these toxicities were grade 1 or 2 and the maximum tolerated dose (MTD) was not determined. Cross-intolerance with imatinib was not evident. ${ }^{14}$

Complete hematologic responses (CHR) were achieved in $92 \%$ of CP-CML, $45 \%$ of AP-CML, $35 \%$ of MBC-CML and $70 \%$ of lymphoid blast crisis (LBC-CML) or Ph+ ALL patients. Major cytogenetic remission (MCyR) were also observed in $45 \%$ of CP-CML, $27 \%$ of AP-CML, 35\% of MBC-CML, and $80 \%$ of LBC-CML or Ph+ ALL patients. Most of these responses occurred in patients receiving doses of 50 to $70 \mathrm{mg}$ twice a day. Responses were seen in patients harboring the Bcr-Abl kinase mutations other than the T315I mutant. ${ }^{14}$

\section{Phase II studies}

As the MTD of dasatinib was not defined in the phase I study, the phase II dose was based on its efficacy, and all patients, regardless of phase of disease, received $70 \mathrm{mg}$ twice daily. The START (Src/Abl Tyrosine kinase inhibition Activity: Research Trials of dasatinib) clinical trials comprised 
four single-arm and one randomized phase II studies: START-C for CP-CML, START-A for AP-CML, START-B for MBC-CML, and START-L for LBC-CML or Ph+ ALL. START-R was a randomized trial of dasatinib and high-dose imatinib for patients with CP-CML after failure on imatinib 400 to $600 \mathrm{mg}$ daily.

A total of 387 imatinib-resistant or -intolerant CP-CML patients were recruited into the START-C study. ${ }^{15}$ With a median follow-up of 15.2 months, the CHR rates were $91 \%$ and MCyR rates were 59\%. A CCyR was attained in 49\% of patients. Loss of MCyR occurred in 3\% of patients. The progression-free survival (PFS) at 15.2 months was $90 \%$ and the overall survival (OS) was $96 \%$. The causes of death in the 14 patients were CML disease $(n=6)$, cardiovascular disease $(\mathrm{n}=2)$, infection $(\mathrm{n}=2)$, central nervous system bleeding $(\mathrm{n}=1)$, and others $(\mathrm{n}=3)$. The toxicity profile was similar to that observed in the phase I study. Approximately $48 \%$ developed a grade 3 or 4 neutropenia or thrombocytopenia, but these were reversible and could be managed with treatment interruption or dose reductions. The most common grade 3 or 4 nonhematologic toxicities were pleural effusion $(6.2 \%)$, dyspnea (5.2\%), diarrhea (2.8\%) and fatigue $(2.1 \%)$. Only $14 \%$ of patients had to discontinue treatment due to drug-related adverse events. These results confirmed that dasatinib was associated with high and durable response rates in imatinib-resistant or -intolerant CP-CML patients and had a favorable toxicity profile. ${ }^{15}$

Dasatinib was also clinically effective in the more advanced phases. The analysis of 107 AP-CML patents enrolled into the START-A trial showed that, at eight months' follow-up, 39\% achieved a CHR and 33\% a MCyR. ${ }^{16}$ The PFS at 10 months was $76 \%$. A total of 14 deaths were reported, of which five were due to disease progression. Myelosuppression was more frequent in the AP-CML patients than in CP-CML. Grade 3 or 4 neutropenia and thrombocytopenia occurred in $76 \%$ and $82 \%$ of patients, respectively. However, no patients had to discontinue treatment due to infection. Nonhematologic adverse events were similar to those of CP-CML. ${ }^{16}$ Responses were also observed in patients with blast crisis but these were generally not durable. A total of $109 \mathrm{MBC}-\mathrm{CML}$ and $48 \mathrm{LBC}-\mathrm{CML}$ were enrolled into the study. ${ }^{17}$ The best CHR rates after a minimum follow-up of 12 months were approximately $28 \%$ in both groups and the best CCyR rates were $26 \%$ and $46 \%$ in the MBC-CML and $\mathrm{LBC}-\mathrm{CML}$ cohorts, respectively. Dasatinib was discontinued in $78 \%$ of MBC-CML and 94\% of LBC-CML patients, and this was due to disease progression in approximately half of the patients. The median PFS was 6.7 months for the MBC-CML and 3.0 months for the LBC-CML patients. There was a high frequency of grade 3 or 4 cytopenias. Compared to the other phases, severe pleural effusion was more frequent in the MBC-CML group, occurring in $15 \%$ of patients. Febrile neutropenia was the most common (15\%) grade 3 or 4 nonhematologic adverse event in the LBC-CML cohort but it did not lead to the discontinuation of dasatinib. ${ }^{17}$

The START phase II studies revealed that dasatinib was effective and safe in imatinib-resistant and -intolerant CML, and the results were comparable with the other second-generation TKIs (Tables 2-4). ${ }^{15-24}$

\section{Phase III dose optimization studies}

Pre-clinical investigations in mice bearing a CML xenograft showed that maximal inhibition of Bcr-Abl and CrkL phosphorylation occurred at approximately three hours after a single oral administration of dasatinib. ${ }^{25}$ This observation, together with the phase I study results, led to the selection of the dasatinib $70 \mathrm{mg}$ twice daily regimen in the phase II studies. However, in the phase I study, similar MCyR rates were also achieved in the once daily regimen. ${ }^{14}$ Longerterm follow-up also suggested that the frequency of pleural effusion was less with the once daily regimen. ${ }^{26}$ Furthermore, due to dose reductions, the median total daily dose in the phase II CP-CML study was $100 \mathrm{mg} .{ }^{15}$ These findings led to the initiation of a phase III study to investigate the efficacy and safety of dasatinib administered as a once or

Table 2 Hematologic and cytogenetic responses in dasatinib phase II trials

\begin{tabular}{lllll}
\hline Stage of disease & $\begin{array}{l}\text { Median duration } \\
\text { of treatment (months) }\end{array}$ & CHR (\%) & MCyR (\%) & CCyR (\%) \\
\hline CP-CML $(n=387)^{15}$ & 13.8 & 91 & 59 & 49 \\
AP-CML $(n=107)^{16}$ & 8.3 & 39 & 33 & 24 \\
MBC-CML $(n=109)^{17}$ & 3.5 & 27 & 33 & 26 \\
LBC-CML $(n=48)^{17}$ & 2.9 & 29 & 52 & 46 \\
\hline
\end{tabular}

Abbreviations: $\mathrm{CHR}$, complete hematological response; $\mathrm{CCyR}$, complete cytogenetic response; $\mathrm{MCyR}$, major cytogenetic response; $\mathrm{CP}-\mathrm{CML}$, chronic phase; $\mathrm{AP}-\mathrm{CML}$, accelerated phase; MBC-CML, myeloid blast crisis; LBC-CML, lymphoid blast crisis. 
Table 3 Hematologic and cytogenetic responses in nilotinib phase II trials

\begin{tabular}{lllll}
\hline Stage of disease & $\begin{array}{l}\text { Median duration } \\
\text { of treatment (months) }\end{array}$ & CHR (\%) & MCyR (\%) & CCyR (\%) \\
\hline CP-CML $(n=321)^{20}$ & 19 & 94 & 59 & 44 \\
AP-CML $(n=138)^{21}$ & 8.9 & 31 & 32 & 20 \\
BC-CML $(n=136)^{22}$ & NR & 11 & 40 & 29 \\
\hline
\end{tabular}

Abbreviation: AP-CML, accelerated phase; BC-CML, blast crisis; CP-CML, chronic phase; CHR, complete hematological response; CCyR, complete cytogenetic response; MCyR, major cytogenetic response; NR, not reported.

twice daily regimen with a total daily dose of $100 \mathrm{mg}$ or $140 \mathrm{mg}$ in CP-CML patients who were resistant or intolerant to imatinib.

Six hundred and seventy CP-CML patients were randomized between four dasatinib treatment arms: $100 \mathrm{mg}$ once daily, $50 \mathrm{mg}$ twice daily, $140 \mathrm{mg}$ once daily, or $70 \mathrm{mg}$ twice daily. ${ }^{27}$ With a minimum follow-up of 24 months and median treatment duration of 22 months, the $\mathrm{CHR}$ rates $(87 \%$ to $92 \%$ ) and CCyR rates (61\% to $63 \%$ ) were comparable in all four groups. Among the patients who achieved a CCyR, the median time to CCyR with dasatinib $100 \mathrm{mg}$ once daily was 13.0 weeks, compared with 12.9 to 13.8 weeks for the other treatment arms. The major molecular response (MMR) rate was $38 \%$ in the four cohorts. The PFS and OS in the $100 \mathrm{mg}$ once daily group at 24 months was $80 \%$ and $91 \%$, respectively. Again, these were comparable to the other arms. Significantly, the incidence of pleural effusion of any grade in the $100 \mathrm{mg}$ once daily treated patients was less than in the other patients $(14 \%$ versus $23 \%-25 \%, \mathrm{p}=0.049)$. Grade 3 or 4 thrombocytopenia was also less frequent in the $100 \mathrm{mg}$ once daily arm (23\% versus $36 \%-41 \%, \mathrm{p}=0.003)$. There was a trend towards a lower incidence of grade 3 or 4 neutropenia in the $100 \mathrm{mg}$ once daily cohort but this was not statistically significant (35\% versus $44 \%-47 \%$, $\mathrm{p}=0.127)$. Treatment with dasatinib $100 \mathrm{mg}$ once daily was also associated with the lowest incidence of treatment interruption (62\% versus $72 \%-79 \%)$, dose reduction $(39 \%$ versus $46 \%-62 \%$ ) and discontinuation due to drug toxicity (12\% versus $16 \%-21 \%) .{ }^{27}$ These findings confirm that the current approved dose of dasatinib $100 \mathrm{mg}$ once daily in CP-CML offers the most favorable long-term benefit-risk assessment. In addition, these observations are further supported by a recent study which shows that transient in vitro inhibition of Bcr-Abl, through high-dose dasatinib pulse therapy, is sufficient to induce irreversible cytotoxicity in CML cells. ${ }^{28}$

The once or twice daily schedule with a total daily dose of $140 \mathrm{mg}$ was also investigated in advanced phase patients. A total of 317 AP-CML patients was assigned randomly to either $140 \mathrm{mg}$ once daily or $70 \mathrm{mg}$ twice daily. ${ }^{29}$ After a minimum follow-up of 24 months, the CHR rates $(47 \%$ versus $52 \%$ ) and MCyR rates (39\% versus $43 \%$ ) rates were similar in the once daily and twice daily arms. The median PFS was comparable in the two groups. Patients in the oncedaily arm had significantly fewer pleural effusions of any grade compared to those in the twice daily arm $(20 \%$ versus $39 \%, \mathrm{p}<0.001)$. The rates of the other nonhematologic and hematologic toxicities were similar between both arms. ${ }^{29}$ There was no appreciable difference in efficacy or treatmentrelated toxicities in the blast crisis-CML (BC-CML) patients who were treated with $140 \mathrm{mg}$ once daily or $70 \mathrm{mg}$ twice daily. ${ }^{30}$ However, there was a suggestion that the once daily dose may improve tolerability as evidenced by a lower incidence of dose reduction ( $4 \%-6 \%$ versus $10 \%-11 \%) .{ }^{30}$

Table 4 Hematologic and cytogenetic responses in bosutinib phase II trials

\begin{tabular}{|c|c|c|c|c|}
\hline Stage of disease & $\begin{array}{l}\text { Median duration } \\
\text { of treatment (months) }\end{array}$ & CHR (\%) & MCyR (\%) & CCyR (\%) \\
\hline $\mathrm{CP}-\mathrm{CML}^{23}$ & 7.3 & $\begin{array}{l}81 \\
\text { (102 evaluable) }\end{array}$ & $\begin{array}{l}47 \\
\text { (I } 46 \text { evaluable) }\end{array}$ & $\begin{array}{l}34 \\
\text { (I46 evaluable) }\end{array}$ \\
\hline $\mathrm{AP}-\mathrm{CML}^{24}$ & 5.5 & $\begin{array}{l}54 \\
\text { (24 evaluable) }\end{array}$ & $\begin{array}{l}47 \\
\text { (30 evaluable) }\end{array}$ & $\begin{array}{l}27 \\
\text { (30 evaluable) }\end{array}$ \\
\hline $\mathrm{BC}-\mathrm{CML}^{24}$ & 2.5 & $\begin{array}{l}36 \\
\text { (14 evaluable) }\end{array}$ & $\begin{array}{l}53 \\
\text { (17 evaluable) }\end{array}$ & $\begin{array}{l}35 \\
\text { (I7 evaluable) }\end{array}$ \\
\hline
\end{tabular}

Abbreviations: AP-CML, accelerated phase; $\mathrm{BC}-\mathrm{CML}$, blast crisis; $\mathrm{CC}$ R, complete cytogenetic response; CP-CML, chronic phase; $\mathrm{CHR}$, complete hematological response; MCyR, major cytogenetic response. 


\section{Predictors of response to dasatinib}

The knowledge that the Bcr-Abl kinase mutations have varying degrees of in vitro sensitivity to dasatinib may allow its use in a rational manner. For example, the highly resistant T315I mutant would be expected to confer refractoriness to dasatinib in patients harboring this mutant. It is also conceivable that patients with mutant clones which have intermediate in vitro resistance may not have an optimal response to dasatinib. In an analysis of 1,043 CP-CML patients enrolled into the phase II and III dasatinib trials, 402 patients had one or more baseline Bcr-Abl kinase mutations. ${ }^{31}$ The T315I mutant was detected in 21 patients and not surprisingly, the majority did not achieve any response with dasatinib. Excluding the T315I mutant, 44 patients had a mutation with a known dasatinib cellular $\mathrm{IC}_{50}$ of more than $3 \mathrm{nM}(\mathrm{Q} 252 \mathrm{H}, \mathrm{E} 255 \mathrm{~K} / \mathrm{V}$, V299L and F317L). Although responses to dasatinib were observed in patients who had any of these five mutations, the frequency of responses was less compared to that of patients who had mutations with a dasatinib cellular $\mathrm{IC}_{50}$ of less than $3 \mathrm{nM}$ or with unknown $\mathrm{IC}_{50}$ (Table 5). ${ }^{31}$

Apart from using existing Bcr-Abl kinase mutations to predict response to dasatinib and other second generation TKI, it may be possible to predict the probability of achieving CCyR using baseline clinical features. A multivariate analysis of 80 imatinib-resistant or -intolerant patients, who were treated with dasatinib $(n=67)$ or nilotinib $(n=13)$ revealed that a low Sokal risk score at diagnosis, the best cytogenetic response on imatinib, the use of growth factor support during imatinib treatment and the time from detection of imatinib failure to commencement of a second generation TKI were predictive factors for achieving CCyR. ${ }^{32}$

Early responses may also be predictive of subsequent responses. In a study of 113 patients (87 CP-CML, 26 AP-CML) who were treated with either dasatinib $(\mathrm{n}=70)$ or nilotinib $(n=43)$, patients who achieved a minor cytogenetic

Table 5 Response rates after at least 24 months of follow-up

\begin{tabular}{llll}
\hline & \multicolumn{2}{l}{ Dasatinib IC } & \\
\cline { 2 - 4 } & $>\mathbf{3} \mathbf{n M}^{\mathrm{a}}$ & $<\mathbf{3} \mathbf{n M}^{\mathrm{b}}$ & $\begin{array}{l}\text { Unknown } \\
(\mathbf{n}=\mathbf{8 3})\end{array}$ \\
\hline CHR (\%) & 82 & 94 & 96 \\
MCyR (\%) & 34 & 58 & 73 \\
CCyR (\%) & 25 & 47 & 54 \\
MMR (\%) & 18 & 34 & 43 \\
\hline
\end{tabular}

Notes: a ${ }^{\text {Excluding }} \mathrm{T} 3 \mathrm{I}$ 5|; ${ }^{\mathrm{b}}$ Excluding patients with a concurrent mutation with dasatinib $\mathrm{IC}_{50}>3 \mathrm{nM}$; 'Excluding patients with a concurrent mutation with known dasatinib $\mathrm{IC}_{50}$. Abbreviations: $\mathrm{CHR}$, complete hematological response; $\mathrm{CCyR}$, complete cytogenetic response; MCyR, major cytogenetic response; MMR, major molecular response. response at three or six months, compared to those who did not, had a significantly higher probability of achieving a MCyR at 12 months. ${ }^{33}$ The molecular response after three months of a second generation TKI was also highly predictive of attaining a MMR. ${ }^{34}$ An analysis of 155 CP-CML patients treated with dasatinib $(n=82)$ or nilotinib $(n=73)$ showed that attaining a BCR-ABL transcript level of less than or equal to $1 \%$ by the International Scale ${ }^{35}$ at three months resulted in an $86 \%$ probability of achieving a MMR at 24 months. This was significantly higher than the probability for those who had a level between $1 \%$ and $10 \%(55 \%$ probability, $\mathrm{p}=0.0003)$ and those with levels more than $10 \%$ (4\% probability, $p<0.0001) .{ }^{34}$ In the group with BCR-ABL transcript levels of more than $10 \%$ by the international scale, those with a level of $50 \%$ or more had a significantly lower probability of attaining a MCyR at 24 months, compared to those with levels between $10 \%$ and $50 \%(11 \%$ versus $56 \%, \mathrm{p}=0.003) .{ }^{34}$

Therefore, pre-existing Bcr-Abl kinase mutations, certain pre-treatment disease features and early cytogenetic and molecular responses are useful in predicting the subsequent clinical responses in patients on dasatinib therapy.

\section{Significance of dasatinib-induced response in CP-CML}

In the first-line treatment of CML with imatinib, the achievement of a MCyR at 12 months and a MMR at 18 months is associated with a significantly lower probability of disease progression at five years. ${ }^{1}$ An analysis of 448 imatinib-resistant or -intolerant CP-CML patients showed that after 12 months of treatment with dasatinib, 57\% achieved a MCyR and 45\% a CCyR. ${ }^{36}$ Achievement of a MCyR at 12 months was associated with a higher 24 -month PFS of $94 \%$, compared to $79 \%$ in those who did not $(\mathrm{p}<0.0001)$. Likewise, patients who achieved a CCyR at 12 months had a higher 24-month PFS than those who did not $(95 \%$ versus $82 \%, p=0.0003) .{ }^{36}$ In a separate evaluation of 1,150 CP-CML patients, $35 \%$ achieved a MMR within a median time of 5.7 months. ${ }^{37} \mathrm{~A}$ landmark analysis showed that achieving a MMR at 12 months conferred a 24-month PFS of $96 \%$ which was significantly higher than not attaining that milestone $(82 \%, \mathrm{p}<0.0001) .{ }^{37}$ This preliminary data suggest that the attainment of a MCyR, CCyR, or MMR at 12 months in dasatinib-treated CP-CML patients may lead to a higher PFS.

\section{Dasatinib in the treatment of $\mathrm{Ph}+$ central nervous system leukemia}

The penetration of imatinib into the cerebrospinal fluid (CSF) is poor, leading to insufficient concentrations for kinase 
inhibition. ${ }^{38}$ Not surprisingly, central nervous system (CNS) leukemic relapses are common in imatinib-treated patients with LBC-CML, MBC-CML, or Ph+ ALL in spite of complete responses in the peripheral blood and bone marrow. ${ }^{39-42}$ A case report of a dasatinib-induced clinical response in $\mathrm{Ph}+$ CNS leukemia led to the further investigation of its utility for these cases. ${ }^{43}$ Using a mouse model, where a human CML tumor cell line was implanted intracranially, treatment with dasatinib significantly increased the median survival time compared to the control and the imatinib-treated groups. ${ }^{44}$ The antitumor activity, as assessed by bioluminescence imaging, was also decreased in the dasatinib-treated group. However, when dasatinib was stopped, the intracranial tumors recurred and all the mice died. Pharmacokinetic analysis also showed that although dasatinib brain concentrations were 12 to 31 times lower than that in the plasma, the levels were adequate to inhibit $50 \%$ of cellular proliferation of the CML cell line in vitro. ${ }^{44}$

A study of 11 patients with $\mathrm{Ph}+\mathrm{CNS}$ leukemia treated with dasatinib revealed that all the patients had a clinical response. ${ }^{44}$ Complete responses were achieved in seven patients, of which four were treated with dasatinib alone. These responses were durable, lasting between three to 12 months. Interestingly, dasatinib-resistant Bcr-Abl kinase mutants (T315I and V299L) were detected in the CSF leukemic blasts of two patients who developed a CNS relapse on dasatinib, suggesting that the selection of a resistant clone, rather than poor CSF penetration, was the cause of relapse. ${ }^{44}$

\section{Dasatinib in children and adolescents}

The role of dasatinib in children and adolescents was investigated in a phase I/II dose-finding study which enrolled 25 patients with $\mathrm{CP}-\mathrm{CML}$, advanced phase $\mathrm{CML}$ or $\mathrm{Ph}+$ acute leukemia, as well as 22 patients with $\mathrm{Ph}$-negative acute leukemia. ${ }^{45}$ The median age was 10 years and all patients were heavily pre-treated. Dasatinib was well tolerated up at to $120 \mathrm{mg} / \mathrm{m}^{2}$ once daily, and the MTD was not reached. CML or $\mathrm{Ph}+$ acute leukemia patients were given a dose of 60 or $80 \mathrm{mg} / \mathrm{m}^{2}$ once daily. Common treatment-related adverse events included nausea, vomiting, diarrhea, headache and rash. Among the 12 CP-CML patients, 83\% achieved a CHR and $67 \%$ a MCyR after a median duration of treatment of 58 days. A CHR rate of $23 \%$ and CCyR rate of $62 \%$ was obtained in the 13 advanced phase or $\mathrm{Ph}+$ acute leukemia patients. The preliminary findings indicate that dasatinib in effective and tolerable in children and adolescents with $\mathrm{Ph}+$ leukemia. ${ }^{45}$

\section{Dasatinib-related toxicities}

Dasatinib is generally well tolerated and its toxicity profile is similar, but not identical, to that of the other TKIs (Tables 6 and 7). However the incidence of cytopenias, pleural effusion and immune-mediated adverse events in patients treated with dasatinb are higher than those on imatinib or nilotinib therapy. This could be due, in part, to dasatinib's higher potency and wider spectrum of kinase inhibition. The fact that there is some differential toxicity between the three drugs may be actually important and useful for a decision on which inhibitor to use or to change to in case of resistance to one of them.

\section{Myelosuppression}

Regardless of the phase of disease, grade 3 or 4 neutropenia and thrombocytopenia occurs more commonly in patients treated with dasatinib than those on imatinib, nilotinib or bosutinib (Table 6). ${ }^{1,15-24}$ This is probably a reflection of its more potent inhibition of Bcr-Abl and Kit. Myelosuppression is generally manageable with dose interruption and reduction. The incidence of grade 3 or 4 cytopenia was also reduced in CP-CML patients who were treated with the $100 \mathrm{mg}$ once

Table 6 Incidence of grade 3 or 4 cytopenias in phase II studies

\begin{tabular}{lllll}
\hline & & CP-CML (\%) & AP-CML (\%) & MBC-CML (\%) \\
\hline Imatinib 400 or $600 \mathrm{mg}$ & Neutropenia & 17 & 58 & 64 \\
once daily $1,18,19$ & Thrombocytopenia & 9 & 43 & 62 \\
Nilotinib $400 \mathrm{mg}$ & Neutropenia & 31 & 42 & 67 \\
twice daily20-22 & Thrombocytopenia & 31 & 41 & 62 \\
Bosutinib $500 \mathrm{mg}$ & Neutropenia & 12 & 28 & 68 \\
once daily 23,24 & Thrombocytopenia & 21 & 62 & 76 \\
Dasatinib $70 \mathrm{mg}$ & Neutropenia & 49 & 76 & 80 \\
twice daily & Thr-17 & 48 & 82 & 82 \\
\hline
\end{tabular}

Abbreviations: AP-CML, accelerated phase; CP-CML, chronic phase; MBC-CML, myeloid blast crisis. 
Table 7 Incidence of selected nonhematologic toxicities (all grades) in chronic-phase chronic myeloid leukemia patients

\begin{tabular}{|c|c|c|c|}
\hline & $\begin{array}{l}\text { Imatinib } 400 \text { mg } \\
\text { once daily }(\%)^{90}\end{array}$ & $\begin{array}{l}\text { Nilotinib } 400 \text { mg } \\
\text { twice daily }(\%)^{20}\end{array}$ & $\begin{array}{l}\text { Dasatinib } 70 \text { mg } \\
\text { twice daily }(\%)^{91}\end{array}$ \\
\hline Peripheral edema & 56 & 7 & 18 \\
\hline Pleural effusion & NR & 2 & 19 \\
\hline Elevated ALT/AST & 43 & $68 / 55$ & $52 / 60$ \\
\hline Elevated serum lipase & NR & 46 & NR \\
\hline Rash & 34 & 31 & 22 \\
\hline Nausea & 44 & 25 & 19 \\
\hline Fatigue & 35 & 20 & 28 \\
\hline Headache & 31 & 18 & 34 \\
\hline Muscle cramps & 38 & NR & NR \\
\hline
\end{tabular}

Abbreviation: NR, not reported.

daily schedule. ${ }^{27}$ Neutropenia can also be overcome with the use of growth factors, thus enabling longer periods of uninterrupted dasatinib treatment. ${ }^{46}$

\section{Pleural effusion}

Dasatinib-related pleural effusion is a frequent adverse event occurring in $7 \%-35 \%$ of patients, with the lowest frequency in CP-CML patients receiving the $100 \mathrm{mg}$ once daily dose and the highest in MBC-CML patients receiving the $70 \mathrm{mg}$ twice daily dose. ${ }^{15-17,26}$ The median time from the start of dasatinib to the development of pleural effusion was 84 days in MBC-CML patients and 315 days in CP-CML patients taking dasatinib $100 \mathrm{mg}$ once daily. ${ }^{17,47}$ Pleural fluid analysis revealed that the majority of cases were exudative in nature with a predominance of lymphocytes..$^{48,49}$ The management of pleural effusion includes dose interruption and reduction, diuretics, corticosteroids and, in severe cases, thoracocentesis. ${ }^{48,49}$ Two separate multivariate analyses identified that certain clinical features were associated with an increased risk of developing pleural effusion. ${ }^{48,50}$ In one study, prior cardiac history, hypertension and a twice-daily dasatinib schedule were independent predictors for the development of pleural effusion. ${ }^{48}$ In the second study, the associated factors were hypercholesterolemia, a history of skin rash on imatinib and a previous history of autoimmune disease. ${ }^{50}$ The latter association and the observations that dasatinib-related pleural effusion has a lymphocyte predominance and is responsive to corticosteroids suggest that the pathogenesis of pleural effusion may be immune-mediated..$^{50}$ The inhibition of PDGFR $\beta$ by dasatinib has also been implicated as a cause of pleural effusion, and it has been shown that PDGFR $\beta$-deficient mouse embryos develop defective blood vessels and edema due to a loss of microvascular pericytes. ${ }^{48,51}$

\section{Dasatinib-induced immune-mediated complications}

Dasatinib is a potent inhibitor of the SFK member, Lck, which is involved in T-cell receptor (TCR) signaling and activation. ${ }^{11,52}$ It has been shown to suppress TCR-mediated signaling, cellular proliferation, cytokine production, in vivo T-cell responses and in vitro natural killer cell cytotoxicity. ${ }^{52-54}$ These findings suggest that patients taking dasatinib may be prone to opportunistic infections due to potential T-cell inhibition, and that dasatinib may, in fact, have a therapeutic role for T-cell mediated immune disorders such as graft-versus-host disease or rheumatoid arthritis. However, dasatinib-induced Lck inhibition has also been implicated in the pathogenesis of autoimmune disorders in two recent case reports. ${ }^{49,55}$ In the first case, the patient presented with respiratory symptoms, diffuse ground glass opacities on thoracic high-resolution computer tomography and appearance of antinuclear and anti-DNA antibodies after 29 days of treatment with dasatinib. ${ }^{49}$ The second case developed fever, arthralgia, pleural effusion, pericardial effusion and autoantibodies after 6 months of dasatinib therapy, suggesting a possibility of dasatinib-induced lupus. ${ }^{55}$ In both cases, the autoantibodies were not present before dasatinib. After cessation of dasatinib, the symptoms were resolved and the autoantibody levels were decreased in the former case and were undetectable in the latter. ${ }^{49,55}$

Another possible mechanism underlying the immunemediated toxicities is the clonal expansion of natural killer/ T-cell lineage large granular lymphocytes (LGL). LGL lymphocytosis has been observed in patients on dasatinib and this correlated with the achievement of complete molecular responses ${ }^{56,57}$ However, patients with LGL lymphocytosis had a high incidence of dasatinib-related adverse events with 
more than 50\% developing pleural effusion, pneumonitis, CMV reactivation, colitis and prolonged fever. ${ }^{57}$

\section{Mechanisms of resistance to dasatinib}

Three major mechanisms have been identified for imatinib resistance. The two most common affect the $B C R-A B L$ gene itself, namely mutations in its tyrosine kinase domain and overexpression of the $\mathrm{Bcr}-\mathrm{Abl}$ protein due to amplification of the $B C R-A B L$ gene. ${ }^{58-62}$ The third mechanism is represented by phenomena which lead to Bcr-Abl-independent resistance. These include upregulation of the ABCB1 or ABCG2 drug efflux pumps, ${ }^{58,63,64}$ downregulation of human organic cation transporter 1 (hOCT1) drug influx transporters, ${ }^{65,66}$ overexpression of Lyn, a Src-family kinase, ${ }^{67}$ and other Bcr-Abl-independent mechanisms. Dasatinib, due to its increased potency and efficacy against most of the imatinibresistant Bcr-Abl kinase mutations, has been able to overcome most forms of imatinib resistance mediated by the former two mechanisms. However, resistance to dasatinib has also become an emerging therapeutic problem.

\section{Dasatinib-resistant Bcr-Abl kinase mutations}

Pre-clinical in vitro and in vivo studies have shown that the T315I mutant is resistant to dasatinib. ${ }^{9,13}$ The dasatinib cellular $\mathrm{IC}_{50}$ for this mutant is more than 250 times higher than that for wild type Bcr-Abl, and kinase activity is not inhibited even in the presence of micromolar concentrations of the drug. ${ }^{9,13}$ Dasatinib treatment did not reduce leukemic burden nor improve survival of mice harboring the T315I mutant. ${ }^{68}$ Co-crystal studies have demonstrated that the T315 is a critical contact residue for dasatinib. ${ }^{69}$ Dasatinib interacts with the side chain of T315 by a hydrogen bond and loss of this hydrogen bond in the T315I mutant, together with an increased steric bulk in this pocket, are the most likely causes of dasatinib resistance. ${ }^{69}$ Not surprisingly, nearly all imatinib-resistant patients with the T315I mutant did not have any clinical response to dasatinib. ${ }^{15-17,31,70}$

Using two different in vitro mutagenesis screening techniques, twelve Bcr-Abl mutants at six sites were identified which conferred in vitro resistance to dasatinib (Table 8). ${ }^{71,72}$ Crystallographic studies have shown that four of these six sites (L248, V299, T315, and F317) are dasatinib contact residues. ${ }^{69}$ Dasatinib makes contact with L248 and G321 by van der Waals interactions and the 2-chlor-6-methyl phenyl ring of dasatinib occupies a hydrophobic pocket composed of
Table 8 Bcr-Abl mutants recovered in the presence of dasatinib and cellular $I C_{50}$

\begin{tabular}{|c|c|c|}
\hline Predicted mutation & Bradeen et $\mathrm{al}^{71}$ & Burgess et $\mathrm{al}^{72}$ \\
\hline L248R & & $+(12)$ \\
\hline L248V & $+(2)$ & \\
\hline Q252H & $+(2)$ & $+(\mathrm{NR})$ \\
\hline E255K & $+(7)$ & $+(9.7)$ \\
\hline V299L & $+(13.4)$ & $+(13.4)$ \\
\hline T3I5A & & $+(93)$ \\
\hline Т3।5I & $+(>250)$ & $+(>750)$ \\
\hline F3I7C & $+(N R)$ & \\
\hline F3I7I & $+(N R)$ & $+(\mathrm{NR})$ \\
\hline F3I7L & $+(9)$ & $+(13.4)$ \\
\hline F3I7S & & $+(\mathrm{NR})$ \\
\hline F3I7V & $+(40)$ & $+(40)$ \\
\hline
\end{tabular}

Notes: () = Fold dasatinib $I C_{50}$ wild type Bcr-Abl.

T315, M290, V299, I313, and A380. ${ }^{69}$ The aromatic ring in the side chain of F317 interacts directly with the pyrimidine and thiazole rings of dasatinib. ${ }^{69}$

Consistent with the in vitro mutagenesis data, the selection of the V299L, T315I, and F317L mutants has been implicated in the emergence of resistance in patients treated with dasatinib..$^{70,73-77}$ Four separate studies have shown that the T315I mutant accounted for $8 \%$ to $70 \%$ of dasatinibresistant mutations ${ }^{31,70,73-75}$ A possible explanation for the wide variation is that the studies with a higher incidence of T315I mutant had a larger proportion of BC-CML and $\mathrm{Ph}+$ ALL patients.

The F317L mutant has intermediate in vitro resistance to dasatinib. ${ }^{13}$ Although CHR were achieved with dasatinib in imatinib-resistant patients harboring this mutant, MCyR were attained in less than $10 \%{ }^{31}$ The F317L mutant was also detected in $12 \%$ to $42 \%$ of patients who developed resistance to dasatinib. ${ }^{31,70,73-75}$ The median OS of patients with the F317L was 19 months from the time of detection and this was similar to that of patients with other mutations. ${ }^{76}$ The survival was dependent on the phase of disease and the two-year OS was 75\% in CP-CML, 50\% in AP-CML, and $20 \%$ in BC-CML patients. $^{76}$

The V299L mutant was detected in $12 \%$ to $25 \%$ of dasatinib-resistant patients. ${ }^{31,73,74}$ This is a novel mutation that had rarely or never been seen in imatinib-resistant patients. $^{74,77}$ The V299L mutant retains in vitro sensitivity to imatinib and nilotinib and clinical responses have been observed in patients treated with these compounds. ${ }^{73,77}$ 
A possible strategy to suppress the outgrowth of resistant clones is the combination of dasatinib or the other Bcr-Abl TKIs with an inhibitor of T315I. SGX393 is an azaindole which inhibits the growth of cells expressing wild type Bcr-Abl and the T315I mutant, as well as other Bcr-Abl mutants at varying concentrations. ${ }^{78}$ SGX393 also reduced CrkL phosphorylation in primary hematopoietic cells from patients harboring the T315I mutant and inhibited growth of T315I-driven tumors in mice. ${ }^{78}$ In addition, the combination of dasatinib and SGX393 completely inhibited the growth of mutant clones at most dose combinations except at intermediate concentrations of dasatinib and SGX393. ${ }^{78}$

\section{Dasatinib cellular influx and efflux}

Multidrug resistance (MDR) due to cross-resistance of mammalian cells to a number of anticancer agents following exposure to one such drug is a well described mechanism of resistance in cancer therapy. This is mediated by an increased expression at the cell surface of the $A B C B 1$ gene product, P-glycoprotein, an energy dependent efflux pump, which reduces intracellular drug concentrations and leads to ineffective levels of the drug reaching its target. ${ }^{79}$ Imatinib is a substrate of $A B C B 1$, and the intracellular levels of imatinib were shown to be significantly lower in $\mathrm{ABCB} 1$-expressing cells. ${ }^{65,80-82}$ However, ABCB1 overexpression has not been reported in patients who are resistant to imatinib. Two other drug transporters, $\mathrm{ABCG} 2$ and hOCT1, have been implicated as possible mechanisms for promoting imatinib resistance. Imatinib has been reported to be a substrate and/or an inhibitor for the ABCG2 drug efflux pump which is overexpressed in many human tumors and also found to be functionally expressed in CML stem cells. ${ }^{64,83,84}$ The drug transporter hOCT1 mediates the active transport of imatinib into cells, and inhibition of hOCT1 decreases the intracellular concentration of imatinib, which may predict for a less favorable molecular response. ${ }^{65,66}$

The cellular influx of dasatinib, on the other hand, is not affected by hOCT1 activity, although its efflux may be mediated by $\mathrm{ABCB} 1$ and $\mathrm{ABCG} 2{ }^{85,86}$ The intracellular concentration of dasatinib was lower in ABCB1-overexpressing cell lines and was increased in the presence of PSC833, a potent $\mathrm{ABCB} 1$ inhibitor ${ }^{85}$ The in vitro concentration of dasatinib required to inhibit phosphorylation of CrkL by $50 \%$ (dasatinib $\left.\mathrm{IC}_{50}\right)$ was higher in the ABCB1-overexpressing cell lines compared to parental cell lines ( $100 \mathrm{nM}$ versus $7 \mathrm{nM})$, and this was reduced to $8 \mathrm{nM}$ with the addition of PSC $833 .{ }^{85}$ Similar results were also seen in the $\mathrm{ABCG} 2$-overexpressing cell lines, suggesting that high levels of $\mathrm{ABCG} 2$ reduced dasatinib intracellular concentration, leading to an increased dasatinib $\mathrm{IC}_{50}$, which can be modulated with ABCG2 inhibitors. ${ }^{85}$

The intracellular concentration of dasatinib in primary cells from CP-CML patients was not significantly decreased with the addition of hOCT1 inhibitors. ${ }^{85,86}$ Dasatinib $\mathrm{IC}_{50}$ was also not significantly different in hOCT1-overexpressing or control cell lines and in cells from patients with high or low hOCT-1 activity. ${ }^{85,86}$ In addition, there was no correlation between the intracellular concentration of dasatinib and dasatinib $\mathrm{IC}_{50}$ in newly diagnosed CP-CML patients. ${ }^{85}$

\section{Disease persistence}

Small numbers of primitive nondividing stem cells have been identified to be refractory to the pro-apoptotic effect of imatinib and conventional chemotherapeutic agents. ${ }^{87}$ The insensitivity of these 'quiescent' cells also has important implications for the management of CML with regards to minimal residual disease and relapse following imatinibinduced response. Although dasatinib significantly inhibited CrkL phosphorylation and caused a reduction in the total number of CD34+CD38-CML cells compared to imatinib, it did not eliminate the most primitive, quiescent fraction. ${ }^{88}$ Recently, the farnesyl transferase inhibitor, BMS-214662 was reported to enhance the cytotoxic effect of imatinib or dasatinib in primary CD34+ CML cells and significantly reduce the numbers of undivided primitive quiescent $\mathrm{CML}$ stem cells, either alone or in combination with imatinib or dasatinib. ${ }^{89}$ This effect was selective and normal stem cells were relatively spared. The cytotoxic action was via apoptosis as evidenced by enhanced caspase-3 activity. ${ }^{89}$ BMS-214662 is currently in phase I trials in acute myeloid leukemia and the possibility of clinical trials in CML is being explored. ${ }^{89}$

\section{Conclusion}

Dasatinib, through its increased potency and ability to bind Bcr-Abl with less stringent conformational requirements, is clinically effective in the treatment of imatinib-resistant CML, leading to durable hematologic and cytogenetic responses and improved PFS and OS. It is generally well tolerated but, due to its wider spectrum of kinase inhibition, certain toxicities occur more frequently than with other TKIs. Dasatinib resistance, due to Bcr-Abl kinase mutation and potentially overexpression of drug efflux transporters, will represent the next therapeutic challenge in CML. Pre-clinical data suggests that combination treatment may prevent the selection of resistant mutants and eradicate primitive stem cells but this will require clinical validation and will represent the next phase of development. 


\section{Disclosure}

Dr Charles Chuah has received honoraria from Bristol-Myers Squibb (BMS) and is on the advisory board of BMS and Novartis Pharma. Prof. Junia Melo has no conflict of interest in this work.

\section{References}

1. Druker BJ, Guilhot F, O'Brien SG, et al. Five-year follow-up of patients receiving imatinib for chronic myeloid leukemia. $N$ Engl J Med. 2006;355(23):2408-2417.

2. Hochhaus A, Druker B, Sawyers C, et al. Favorable long-term follow-up results over 6 years for response, survival, and safety with imatinib mesylate therapy in chronic-phase chronic myeloid leukemia after failure of interferon-alpha treatment. Blood. 2008;111(3):1039-1043.

3. Mauro MJ. Defining and managing imatinib resistance. Hematology Am Soc Hematol Educ Program. 2006;219-225.

4. Martin GS. The road to Src. Oncogene. 2004;23(48):7910-7917.

5. Dorsey JF, Jove R, Kraker AJ, Wu J. The pyrido[2,3-d]pyrimidine derivative PD180970 inhibits p210Bcr-Abl tyrosine kinase and induces apoptosis of K562 leukemic cells. Cancer Res. 2000;60(12): 3127-3131.

6. La Rosee P, Corbin AS, Stoffregen EP, Deininger MW, Druker BJ. Activity of the Bcr-Abl kinase inhibitor PD180970 against clinically relevant Bcr-Abl isoforms that cause resistance to imatinib mesylate (Gleevec, STI571). Cancer Res. 2002;62(24):7149-7153.

7. Golas JM, Arndt K, Etienne C, et al. SKI-606, a 4-anilino-3quinolinecarbonitrile dual inhibitor of Src and $\mathrm{Abl}$ kinases, is a potent antiproliferative agent against chronic myelogenous leukemia cells in culture and causes regression of K562 xenografts in nude mice. Cancer Res. 2003;63(2):375-381.

8. Warmuth M, Simon N, Mitina O, et al. Dual-specific Src and Abl kinase inhibitors, PP1 and CGP76030, inhibit growth and survival of cells expressing imatinib mesylate-resistant Bcr-Abl kinases. Blood. 2003;101(2):664-672.

9. Shah NP, Tran C, Lee FY, Chen P, Norris D, Sawyers CL. Overriding imatinib resistance with a novel ABL kinase inhibitor. Science. 2004;305(5682):399-401.

10. O'Hare T, Pollock R, Stoffregen EP, et al. Inhibition of wild-type and mutant Bcr-Abl by AP23464, a potent ATP-based oncogenic protein kinase inhibitor: implications for CML. Blood. 2004;104(8):2532-2539.

11. Lombardo LJ, Lee FY, Chen P, et al. Discovery of N-(2-chloro6-methyl- phenyl)-2-(6-(4-(2-hydroxyethyl)- piperazin-1-yl)-2methylpyrimidin-4-ylamino)thiazole-5-carboxamide (BMS-354825), a dual Src/Abl kinase inhibitor with potent antitumor activity in preclinical assays. J Med Chem. 2004;47(27):6658-6661.

12. Rix U, Hantschel O, Durnberger G, et al. Chemical proteomic profiles of the BCR-ABL inhibitors imatinib, nilotinib, and dasatinib reveal novel kinase and nonkinase targets. Blood. 2007;110(12): 4055-4063.

13. O'Hare T, Walters DK, Stoffregen EP, et al. In vitro activity of Bcr-Abl inhibitors AMN107 and BMS-354825 against clinically relevant imatinib-resistant Abl kinase domain mutants. Cancer Res. 2005;65(11):4500-4505.

14. Talpaz M, Shah NP, Kantarjian H, et al. Dasatinib in imatinibresistant Philadelphia chromosome-positive leukemias. $N$ Engl J Med. 2006;354(24):2531-2541.

15. Hochhaus A, Baccarani M, Deininger M, et al. Dasatinib induces durable cytogenetic responses in patients with chronic myelogenous leukemia in chronic phase with resistance or intolerance to imatinib. Leukemia. 2008;22(6):1200-1206.

16. Guilhot F, Apperley J, Kim DW, et al. Dasatinib induces significant hematologic and cytogenetic responses in patients with imatinibresistant or -intolerant chronic myeloid leukemia in accelerated phase. Blood. 2007;109(10):4143-4150.
17. Cortes J, Kim DW, Raffoux E, et al. Efficacy and safety of dasatinib in imatinib-resistant or -intolerant patients with chronic myeloid leukemia in blast phase. Leukemia. 2008;22(12):2176-2183.

18. Talpaz M, Silver RT, Druker BJ, et al. Imatinib induces durable hematologic and cytogenetic responses in patients with accelerated phase chronic myeloid leukemia: results of a phase 2 study. Blood. 2002;99(6):1928-1937.

19. Sawyers CL, Hochhaus A, Feldman E, et al. Imatinib induces hematologic and cytogenetic responses in patients with chronic myelogenous leukemia in myeloid blast crisis: results of a phase II study. Blood. 2002;99(10):3530-3539.

20. Kantarjian HM, Giles F, Bhalla KN, et al. Nilotinib in chronic myeloid leukemia patients in chronic phase (CMLCP) with imatinib resistance or intolerance: 2-year follow-up results of a phase 2 study. ASH Annual Meeting Abstracts. 2008;112(11):3238.

21. le Coutre PD, Giles F, Hochhaus A, et al. Nilotinib in chronic myeloid leukemia patients in accelerated phase (CML-AP) with imatinib resistance or intolerance: 2-year follow-up results of a phase 2 study. ASH Annual Meeting Abstracts. 2008;112(11):3229.

22. Giles FJ, Larson RA, Kantarjian HM, et al. Nilotinib in patients with Philadelphia chromosome-positive chronic myelogenous leukemia in blast crisis (CML-BC) who are resistant or intolerant to imatinib. J Clin Oncol (Meeting Abstracts). 2008;26(15_suppl):7017.

23. Cortes J, Kantarjian HM, Kim D, et al. Efficacy and safety of bosutinib (SKI-606) in patients with chronic phase (CP) $\mathrm{Ph}+$ chronic myelogenous leukemia (CML) with resistance or intolerance to imatinib. ASH Annual Meeting Abstracts. 2008;112(11):1098.

24. Passerini CG, Pogliani EM, Baccarani M, et al. Bosutinib (SKI-606) demonstrates clinical activity and is well tolerated in patients with $\mathrm{AP}$ and $\mathrm{BP} \mathrm{CML}$ and Ph+ ALL. ASH Annual Meeting Abstracts. 2008;112(11):1101.

25. Luo FR, Yang Z, Camuso A, et al. Dasatinib (BMS-354825) pharmacokinetics and pharmacodynamic biomarkers in animal models predict optimal clinical exposure. Clin Cancer Res. 2006;12(23):7180-7186.

26. Shah NP, Kantarjian HM, Kim DW, et al. Intermittent target inhibition with dasatinib $100 \mathrm{mg}$ once daily preserves efficacy and improves tolerability in imatinib-resistant and -intolerant chronic-phase chronic myeloid leukemia. J Clin Oncol. 2008;26(19):3204-3212.

27. Shah NP, Kim DW, Kantarjian HM, et al. Dasatinib Dose-Optimization in Chronic Phase Chronic Myeloid Leukemia (CML-CP): Two-year data from CA180-034 show equivalent long-term efficacy and improved safety with $100 \mathrm{mg}$ once daily dose. ASH Annual Meeting Abstracts. 2008;112(11):3225.

28. Shah NP, Kasap C, Weier C, et al. Transient potent BCR-ABL inhibition is sufficient to commit chronic myeloid leukemia cells irreversibly to apoptosis. Cancer Cell. 2008;14(6):485-493.

29. Kantarjian HM, Kim DW, Dorlhiac-Llacer P, et al. Dasatinib $140 \mathrm{mg}$ once daily (QD) demonstrates equivalent efficacy and improved safety compared with $70 \mathrm{mg}$ twice daily (BID) in patients with accelerated phase chronic myeloid leukemia (CML-AP): 2-year follow-up data from CA180-035. ASH Annual Meeting Abstracts. 2008;112(11):3224.

30. Saglio G, Kantarjian HM, Hochhaus A, et al. Dasatinib $140 \mathrm{mg}$ once daily (QD) demonstrates equivalent efficacy and improved safety compared with $70 \mathrm{mg}$ twice daily (BID) in patients with chronic myeloid leukemia in blast phase (CML-BP): 2-year data from CA180-035. ASH Annual Meeting Abstracts. 2008;112(11):3226.

31. Muller MC, Cortes J, Kim DW, et al. Dasatinib efficacy in patients with chronic myeloid leukemia in chronic phase (CML-CP) and pre-existing BCR-ABL mutations. ASH Annual Meeting Abstracts. 2008;112(11):449.

32. Milojkovic D, Bua M, Apperley JF, et al. Prediction of cytogenetic response to second generation TKI therapy in CML chronic phase patients who have failed imatinib therapy and early identification of factors that influence survival. ASH Annual Meeting Abstracts. 2008;112(11):332.

33. Tam CS, Kantarjian H, Garcia-Manero G, et al. Failure to achieve a major cytogenetic response by 12 months defines inadequate response in patients receiving nilotinib or dasatinib as second or subsequent line therapy for chronic myeloid leukemia. Blood. 2008;112(3):516-518. 
34. Branford S, Lawrence R, Fletcher L, Field C, Rudzki Z, Hughes T. The initial molecular response of chronic phase CML patients treated with second generation $\mathrm{ABL}$ inhibitor therapy after imatinib failure can predict inadequate response and provide indications for rational mutation screening. ASH Annual Meeting Abstracts. 2008;112(11):331.

35. Branford S, Cross NC, Hochhaus A, et al. Rationale for the recommendations for harmonizing current methodology for detecting BCR-ABL transcripts in patients with chronic myeloid leukaemia Leukemia. 2006;20(11):1925-1930.

36. Baccarani M, Rosti G, Saglio G, et al. Dasatinib time to and durability of major and complete cytogenetic response (MCyR and CCyR) in patients with chronic myeloid leukemia in chronic phase (CML-CP). ASH Annual Meeting Abstracts. 2008;112(11):450.

37. Hochhaus A, Muller MC, Radich J, et al. Dasatinib-associated major molecular responses are rapidly achieved in patients with chronic myeloid leukemia in chronic phase (CML-CP) following resistance, suboptimal response, or intolerance on imatinib. ASH Annual Meeting Abstracts. 2008;112(11):1095.

38. Takayama N, Sato N, O'Brien SG, Ikeda Y, Okamoto S. Imatinib mesylate has limited activity against the central nervous system involvement of Philadelphia chromosome-positive acute lymphoblastic leukaemia due to poor penetration into cerebrospinal fluid. Br J Haematol. 2002;119(1):106-108.

39. Pfeifer H, Wassmann B, Hofmann WK, et al. Risk and prognosis of central nervous system leukemia in patients with Philadelphia chromosome-positive acute leukemias treated with imatinib mesylate. Clin Cancer Res. 2003;9(13):4674-4681.

40. Bujassoum S, Rifkind J, Lipton JH. Isolated central nervous system relapse in lymphoid blast crisis chronic myeloid leukemia and acute lymphoblastic leukemia in patients on imatinib therapy. Leuk Lymphoma. 2004;45(2):401-403.

41. Leis JF, Stepan DE, Curtin PT, et al. Central nervous system failure in patients with chronic myelogenous leukemia lymphoid blast crisis and Philadelphia chromosome positive acute lymphoblastic leukemia treated with imatinib (STI-571). Leuk Lymphoma. 2004;45(4):695-698.

42. Matsuda M, Morita Y, Shimada T, et al. Extramedullary blast crisis derived from 2 different clones in the central nervous system and neck during complete cytogenetic remission of chronic myelogenous leukemia treated with imatinib mesylate. Int J Hematol. 2005;81(4) 307-309.

43. Abdelhalim A, Barcos M, Block AW, et al. Remission of Philadelphia chromosome-positive central nervous system leukemia after dasatinib therapy. Leuk Lymphoma. 2007;48(5):1053-1056.

44. Porkka K, Koskenvesa P, Lundan T, et al. Dasatinib crosses the blood-brain barrier and is an efficient therapy for central nervous system Philadelphia chromosome-positive leukemia. Blood. 2008;112(4):1005-1012.

45. Zwaan CM, Rizzari C, van der Velden VHJ, et al. Dasatinib in children and adolescents with relapsed or refractory leukemia: interim results of the CA180-018 phase i study from the ITCC consortium. ASH Annual Meeting Abstracts. 2008;112(11):3241.

46. Quintas-Cardama A, Kantarjian HM, Nicaise C, et al. Cytopenias in patients (pts) with chronic myelogenous leukemia (CML) in chronic phase $(\mathrm{CP})$ treated with dasatinib (SPRYCEL $(\mathrm{R}))$ : clinical features and management, including outcome after hematopoietic growth factor therapy. ASH Annual Meeting Abstracts. 2006;108(11):2163.

47. Porkka K, Khoury HJ, Paquette R, et al. Dasatinib $100 \mathrm{mg}$ once daily (QD) maintains long-term efficacy and minimizes the occurrence of pleural effusion: an analysis of 24-month data in patients with resistance, suboptimal response, or intolerance to imatinib (CA180-034). ASH Annual Meeting Abstracts. 2008;112(11):3242.

48. Quintas-Cardama A, Kantarjian H, O'Brien S, et al. Pleural effusion in patients with chronic myelogenous leukemia treated with dasatinib after imatinib failure. J Clin Oncol. 2007;25(25):3908-3914.

49. Bergeron A, Rea D, Levy V, et al. Lung abnormalities after dasatinib treatment for chronic myeloid leukemia: a case series. Am J Respir Crit Care Med. 2007;176(8):814-818.
50. de Lavallade H, Punnialingam S, Milojkovic D, et al. Pleural effusions in patients with chronic myeloid leukaemia treated with dasatinib may have an immune-mediated pathogenesis. Br J Haematol. 2008;141(5):745-747.

51. Lindahl P, Johansson BR, Leveen P, Betsholtz C. Pericyte loss and microaneurysm formation in PDGF-B-deficient mice. Science. 1997;277(5323):242-245.

52. Schade AE, Schieven GL, Townsend R, et al. Dasatinib, a small-molecule protein tyrosine kinase inhibitor, inhibits T-cell activation and proliferation. Blood. 2008;111(3):1366-1377.

53. Blake SJ, Bruce LA, Fraser CK, Hayball JD, Hughes TP. Dasatinib suppresses in vitro natural killer cell cytotoxicity. Blood. 2008;111(8): 4415-4416.

54. Blake S, Hughes TP, Mayrhofer G, Lyons AB. The Src/ABL kinase inhibitor dasatinib (BMS-354825) inhibits function of normal human T-lymphocytes in vitro. Clin Immunol. 2008;127(3):330-339.

55. Rea D, Bergeron A, Fieschi C, Bengoufa D, Oksenhendler E, Dombret H. Dasatinib-induced lupus. Lancet. 2008;372(9640):713-714.

56. Kim DH, Kamel-Reid S, Chang H, et al. Natural killer or natural killer/T cell lineage large granular lymphocytosis associated with dasatinib therapy for Philadelphia chromosome positive leukemia. Haematologica. 2009;94(1):135-139.

57. Mustjoki S, Ekblom M, Arstila TP, et al. Clonal expansion of T/NK-cells during tyrosine kinase inhibitor dasatinib therapy. $A S H$ Annual Meeting Abstracts. 2008;112(11):573.

58. Mahon FX, Deininger MW, Schultheis B, et al. Selection and characterization of BCR-ABL positive cell lines with differential sensitivity to the tyrosine kinase inhibitor STI571: diverse mechanisms of resistance. Blood. 2000;96(3):1070-1079.

59. Gorre ME, Mohammed M, Ellwood K, et al. Clinical resistance to STI-571 cancer therapy caused by BCR-ABL gene mutation or amplification. Science. 2001;293(5531):876-880.

60. Branford S, Rudzki Z, Walsh S, et al. High frequency of point mutations clustered within the adenosine triphosphate-binding region of BCR/ $\mathrm{ABL}$ in patients with chronic myeloid leukemia or Ph-positive acute lymphoblastic leukemia who develop imatinib (STI571) resistance. Blood. 2002;99(9):3472-3475.

61. Shah NP, Nicoll JM, Nagar B, et al. Multiple BCR-ABL kinase domain mutations confer polyclonal resistance to the tyrosine kinase inhibitor imatinib (STI571) in chronic phase and blast crisis chronic myeloid leukemia. Cancer Cell. 2002;2(2):117.

62. von Bubnoff N, Schneller F, Peschel C, Duyster J. BCR-ABL gene mutations in relation to clinical resistance of Philadelphiachromosome-positive leukaemia to STI571: a prospective study. Lancet. 2002;359(9305):487-491

63. le Coutre P, Tassi E, Varella-Garcia M, et al. Induction of resistance to the Abelson inhibitor STI571 in human leukemic cells through gene amplification. Blood. 2000;95(5):1758-1766.

64. Jordanides NE, Jorgensen HG, Holyoake TL, Mountford JC. Functional ABCG2 is over-expressed on primary CML CD34+ cells and is inhibited by imatinib mesylate. Blood. 2006;108(4):1370-1373.

65. Thomas J, Wang L, Clark RE, Pirmohamed M. Active transport of imatinib into and out of cells: implications for drug resistance. Blood. 2004;104(12):3739-3745.

66. White DL, Saunders VA, Dang P, et al. OCT-1 mediated influx is a key determinant of the intracellular uptake of imatinib but not nilotinib (AMN107); reduced OCT-1 activity is the cause of low in vitro sensitivity to imatinib. Blood. 2006;108(2):697-704.

67. Donato NJ, Wu JY, Stapley J, et al. BCR-ABL independence and LYN kinase overexpression in chronic myelogenous leukemia cells selected for resistance to STI571. Blood. 2003;101(2):690-698.

68. Shah NP, Sawyers CL. Mechanisms of resistance to STI571 in Philadelphia chromosome-associated leukemias. Oncogene. 2003; 22(47):7389-7395.

69. Tokarski JS, Newitt JA, Chang CY, et al. The structure of dasatinib (BMS-354825) bound to activated ABL kinase domain elucidates its inhibitory activity against imatinib-resistant ABL mutants. Cancer Res. 2006;66(11):5790-5797. 
70. Soverini S, Colarossi S, Gnani A, et al. Resistance to dasatinib in Philadelphia-positive leukemia patients and the presence or the selection of mutations at residues 315 and 317 in the BCR-ABL kinase domain. Haematologica. 2007;92(3):401-404.

71. Bradeen HA, Eide CA, O'Hare T, et al. Comparison of imatinib, dasatinib (BMS-354825), and nilotinib (AMN107) in an n-ethyl-nnitrosourea (ENU)-based mutagenesis screen: high efficacy of drug combinations. Blood. 2006;108(7):2332-2338.

72. Burgess MR, Skaggs BJ, Shah NP, Lee FY, Sawyers CL. Comparative analysis of two clinically active BCR-ABL kinase inhibitors reveals the role of conformation-specific binding in resistance. Proc Natl Acad Sci U S A. 2005;102(9):3395-3400.

73. Shah NP, Skaggs BJ, Branford S, et al. Sequential ABL kinase inhibitor therapy selects for compound drug-resistant BCR-ABL mutations with altered oncogenic potency. J Clin Invest. 2007;117(9):2562-2569.

74. Cortes J, Jabbour E, Kantarjian H, et al. Dynamics of BCR-ABL kinase domain mutations in chronic myeloid leukemia after sequential treatment with multiple tyrosine kinase inhibitors. Blood. 2007;110(12):4005-4011.

75. Khorashad JS, Milojkovic D, Mehta P, et al. In vivo kinetics of kinase domain mutations in CML patients treated with dasatinib after failing imatinib. Blood. 2008;111(4):2378-2381.

76. Jabbour E, Kantarjian HM, Jones D, et al. Characteristics and outcome of chronic myeloid leukemia patients with F317L BCR-ABL kinase domain mutation after therapy with tyrosine kinase inhibitors. Blood. 2008;112(13):4839-4842.

77. Jabbour E, Kantarjian HM, Jones D, Burton E, Cortes J. Clinical characteristics and outcome of patients (pts) with V299L BCRABL kinase domain (KD) mutation after therapy with tyrosine kinase inhibitors (TKIs). ASH Annual Meeting Abstracts. 2008;112(11):1105.

78. O'Hare T, Eide CA, Tyner JW, et al. SGX393 inhibits the CML mutant Bcr-AblT315I and preempts in vitro resistance when combined with nilotinib or dasatinib. Proc Natl Acad Sci U S A. 2008;105(14): 5507-5512.

79. Gottesman MM, Fojo T, Bates SE. Multidrug resistance in cancer: role of ATP-dependent transporters. Nat Rev Cancer. 2002;2(1):48-58.

80. Hegedus T, Orfi L, Seprodi A, Varadi A, Sarkadi B, Keri G. Interaction of tyrosine kinase inhibitors with the human multidrug transporter proteins, MDR1 and MRP1. Biochim Biophys Acta. 2002; $1587(2-3): 318-325$.
81. Widmer N, Colombo S, Buclin T, Decosterd LA. Functional consequence of MDR1 expression on imatinib intracellular concentrations. Blood. 2003;102(3):1142.

82. Illmer T, Schaich M, Platzbecker U, et al. P-glycoprotein-mediated drug efflux is a resistance mechanism of chronic myelogenous leukemia cells to treatment with imatinib mesylate. Leukemia. 2004;18(3):401-408.

83. Burger H, van Tol H, Boersma AW, et al. Imatinib mesylate (STI571) is a substrate for the breast cancer resistance protein (BCRP)/ABCG2 drug pump. Blood. 2004;104(9):2940-2942.

84. Burger $\mathrm{H}$, van Tol H, Brok M, et al. Chronic imatinib mesylate exposure leads to reduced intracellular drug accumulation by induction of the ABCG2 (BCRP) and ABCB1 (MDR1) drug transport pumps. Cancer Biol Ther. 2005;4(7):747-752.

85. Hiwase DK, Saunders V, Hewett D, et al. Dasatinib cellular uptake and efflux in chronic myeloid leukemia cells: therapeutic implications. Clin Cancer Res. 2008;14(12):3881-3888.

86. Giannoudis A, Davies A, Lucas CM, Harris RJ, Pirmohamed M, Clark RE. Effective dasatinib uptake may occur without human organic cation transporter 1 (hOCT1): implications for the treatment of imatinib-resistant chronic myeloid leukemia. Blood. 2008;112(8): 3348-3354.

87. Graham SM, Jorgensen HG, Allan E, et al. Primitive, quiescent, Philadelphia-positive stem cells from patients with chronic myeloid leukemia are insensitive to STI571 in vitro. Blood. 2002;99(1): 319-325.

88. Copland M, Hamilton A, Elrick LJ, et al. Dasatinib (BMS-354825) targets an earlier progenitor population than imatinib in primary CML but does not eliminate the quiescent fraction. Blood. 2006;107(11): 4532-4539.

89. Copland M, Pellicano F, Richmond L, et al. BMS-214662 potently induces apoptosis of chronic myeloid leukemia stem and progenitor cells and synergizes with tyrosine kinase inhibitors. Blood. 2008;111(5):2843-2853.

90. O'Brien SG, Guilhot F, Larson RA, et al. Imatinib compared with interferon and low-dose cytarabine for newly diagnosed chronic-phase chronic myeloid leukemia. N Engl J Med. 2003;348(11):994-1004.

91. Hochhaus A, Kantarjian HM, Baccarani M, et al. Dasatinib induces notable hematologic and cytogenetic responses in chronic-phase chronic myeloid leukemia after failure of imatinib therapy. Blood. 2007;109(6):2303-2309.
OncoTargets and Therapy

\section{Publish your work in this journal}

OncoTargets and Therapy is an international, peer-reviewed, open access journal focusing on the pathological basis of all cancers, potential targets for therapy and treatment protocols employed to improve the management of cancer patients. The journal also focuses on the impact of management programs and new therapeutic agents and protocols on

\section{Dovepress}

patient perspectives such as quality of life, adherence and satisfaction The manuscript management system is completely online and includes a very quick and fair peer-review system, which is all easy to use. Visit http://www.dovepress.com/testimonials.php to read real quotes from published authors. 